\title{
Efecto de la Curvatura de la Superficie en la Adsorción de Gases Simples sobre Materiales Carbonosos usando la Teoría del Funcional de la Densidad (DFT)
}

\author{
Alberto G. Albesa, Alejandro Filippín y José L. Vicente \\ Universidad Nacional de La Plata, Facultad de Ciencias Exactas, Depto. de Química, INIFTA \\ (CICPBA, CONICET), Casilla de Correo 16, Sucursal 4, (B1904DPI), La Plata-Argentina \\ (email: albesa@inifta.unlp.edu.ar)
}

\begin{abstract}
Resumen
En el presente trabajo se realizaron cálculos de la adsorción de nitrógeno, metano e hidrógeno sobre superficies carbonosas que poseen distintas curvaturas. Se utilizó la teoría del funcional de la densidad (DFT) mediante el funcional PW91PW91 junto a un conjunto de bases 6-31G (d, p). Los resultados obtenidos fueron ajustados al potencial de Lennard-Jones 12-6 para obtener los parámetros de interacción. Los parámetros que resultan de ajustar un potencial 12-6 de LennardJones no muestran ninguna dependencia importante con la curvatura de los nanotubos en el rango considerado
\end{abstract}

Palabras clave: metano, nitrógeno, adsorción de gases, nanotubos, materiales carbonosos

\section{Effects of the Surface Curvature on the Adsorption of Simple Gases over Carbonaceous Materials using the Density Functional Theory (DFT)}

\begin{abstract}
In this paper calculations of the adsorption of nitrogen, methane and hydrogen on carbonaceous surfaces that have different curvatures are presented. The density functional theory (DFT) was used including a functional PW91PW91 along with a set of 6-31G basis (d, p). The results were adjusted to the potential of 12-6 Lennard-Jones to get the interaction parameters. The parameters of the 12-6 Lennard-Jones potential show no important dependency on the curvature of the nanotubes in the range considered in this study.
\end{abstract}

Keywords: methane, nitrogen, gas adsorption, nanotubes, carbonaceous materials 


\section{INTRODUCCIÓN}

Los nanotubos de carbón y los materiales carbonosos micro porosos en general presentan propiedades interesantes desde el punto de vista tecnológico, ya sea como medio para almacenamiento de hidrógeno y metano (Bhatia y Myers, 2006), que son fuentes de energía ambientalmente favorables, o bien como membranas para la separación de gases como oxígeno y nitrógeno (Arora y Sandler,2007). Las propiedades adsortivas de los nanotubos y fullerenos difieren de otros carbones grafíticos debido a la curvatura (es decir, ángulos en enlace del C-C-C) de la superficie del carbón. Los átomos de carbono deben adoptar una hibridación cuasi-sp2 debido a su estructura altamente curvada. El grado de hibridación del orbital de valencia depende del radio del material: un radio grande conduce a una hibridación cerca de $\mathrm{sp}^{2}$ puro mientras que un radio pequeño a una $\mathrm{sp}^{3}$ (Niyogi et al., 2002).

Para describir los fenómenos que ocurren a nivel molecular se emplean simulaciones, ya sea Monte Carlo Gran Canónica (Albesa et al., 2008), para estudiar la adsorción en equilibrio, o Dinámica Molecular (Cheng et al., 2005) cuando interesan las propiedades de transporte fuera del equilibrio. Sin embargo, la precisión de tales descripciones depende, en gran medida, de los modelos de potencial empleados, utilizándose normalmente el potencial 12-6 de Lennard-Jones, con los parámetros del grafito, o el potencial de Crowell-Brown, que no tienen en cuenta la curvatura de la superficie (Wang y Johnson,1999). Este potencial deberá identificar el punto de equilibrio y brindar una buena aproximación alrededor del mismo, al menos hasta el punto de inflexión de la curva. Para lograr esto se debe contar con una buena descripción de los enlaces moleculares, pues estas aproximaciones podrían despreciar el efecto de la diferencia de hibridación de los orbitales atómicos de los carbonos del sustrato, inducida por la curvatura de la superficie. A tal fin, se derivaron campos de fuerza (Kostov et al., 2002) en donde el efecto de la curvatura fue tenido en cuenta, sin embargo, los parámetros calculados para estos campos sobre estimaban las interacciones con los nanotubos de menor radio al considerar como modelo una molécula con radicales libres.

En el presente trabajo se realizan cálculos teóricos a nivel DFT para moléculas de metano, nitrógeno e hidrógeno sobre la superficie de distintos nanotubos, simulados mediante la deformación de una molécula de coroneno. Para la selección de los adsorbatos (nitrógeno, hidrógeno y metano) se tomó en cuenta que, para los dos primeros, existen alguna información previa en la literatura que permite establecer comparaciones, el nitrógeno es interesante en los procesos de separación de gases, y el metano es una fuente de energía menos compleja que el hidrógeno, para su almacenamiento en nanotubos (Bhatia y Myers, 2006; Zuttel, 2003).

\section{METODOLOGÍA}

\section{Modelo del sustrato}

El modelo utilizado para simular la superficie del sustrato es una molécula de coroneno, tomada a partir de la superficie de un nanotubo, tal como se muestra en la figura 1 . El diámetro $D$ de un nanotubo está dado a través de los índices $(m, n)$, llamados índices de Hamada, en la siguiente forma:

$D_{t}=\frac{a}{\pi} \sqrt{m^{2}+n^{2}+n m}$

Siendo $a=2.46 \AA$ en el presente trabajo se utilizaron nanotubos del tipo 'armchair', en los cuales $m=$ $n$, pues existen evidencias que la fisisorción de gases simples es independiente de la quiralidad (Vilaplana, 2005), de esta forma el diámetro queda definido con un único parámetro $n$. Con el objeto de compararlos posteriormente con resultados experimentales obtenidos en nuestro laboratorio, se seleccionó un rango de diámetros con valores que correspondieran a los nanotubos de simple pared que se obtienen a través de los métodos de producción mas confiables, puesto que cuando los diámetros son grandes en realidad muchas veces se está en presencia de casos de doble pared (Colomer et al., 1999). De manera que se tomaron los casos $(3,3) ;(7,7) ;(15,15)$, que corresponden a diámetros de $4.07 \AA, 9.49 \AA$ y $20.34 \AA$ respectivamente. 


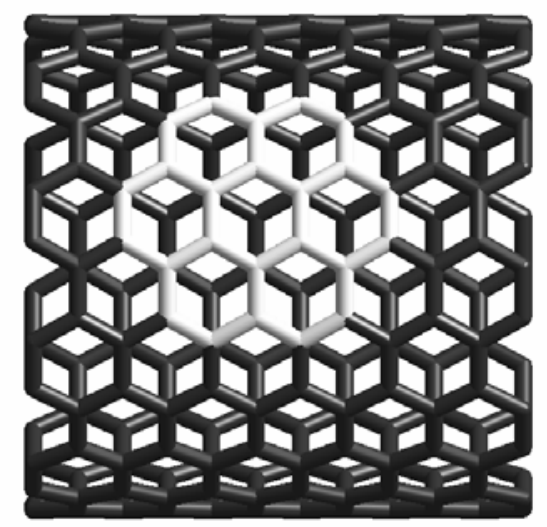

Fig 1: Modelo del racimo de carbono utilizado

\section{Métodos computacionales}

Para determinar las curvas de energía potencial se utilizó la teoría del funcional de la densidad (DFT) por su bajo costo computacional y su precisión respecto a otros métodos ab initio. Se adoptó el funcional PW91PW91 (Wang y Perdew, 1991) junto a un conjunto de bases 6-31G (d, p) empleando el software Gaussian 03 (Frisch et al., 2003). Se utilizó este funcional por brindar buenos resultados en estructuras moleculares de grafito (Albesa y Vicente, 2008). Los errores por la superposición de bases se corrigieron mediante el método de Counterpoise (Boys y Bernardi, 1970).La energía de interacción viene dada por

$\mathrm{E}_{\text {(interacción) }}=\mathrm{E}_{\text {(complejo) }}-\mathrm{E}_{\text {(infinito) }}$

Siendo $\mathrm{E}_{\text {(infinito) }}$ la energía cuando la molécula de coroneno y la molécula del adsorbato se encuentran a una distancia mayor que $350 \AA$. Las moléculas de hidrógeno y nitrógeno se colocaron en forma paralela a la superficie del coroneno en todos los puntos. No se consideró la relajación en la geometría del adsorbato debido a que su efecto sobre la energía total de interacción es despreciable. Los parámetros del potencial 12-6 de Lennard-Jones se calcularon a través de un ajuste con las curvas obtenidas.

\section{RESULTADOS Y DISCUSIÓN}

Los resultados de los cálculos se muestran en las figuras 2-4. En la curva correspondiente al hidrógeno se observa que no hay grandes cambios de la energía de interacción en función del radio del nanotubo y que la energía de interacción es muy débil, al menos en su cara externa, dificultando el uso de estos sustratos (nanotubos cerrados) para su almacenamiento. Para el metano y el nitrógeno, el potencial menos atractivo corresponde a nanotubos de diámetro intermedio; siendo la energía de interacción similar para los potenciales en $(3,3)$ y $(15,15)$.

Cabe destacar que en todas las curvas de energía potencial la posición del mínimo casi no varía y que la gráfica correspondiente al nanotubo de mayor diámetro es la mas abierta, extendiéndose fuera de la superficie.

Experimentalmente, la energía de interacción en nanotubos con hidrógeno es $3.3 \mathrm{KJ} / \mathrm{mol}$ (Okamoto y Miyamoto, 2001), con nitrógeno $10.06 \mathrm{KJ} / \mathrm{mol}$ (Wei et al., 2003) y con metano $10.5 \mathrm{KJ} / \mathrm{mol}$ (Talapatra y Migone, 2002). Si bien, como en la mayoría de los cálculos GGA, las energías son inferiores a las experimentales, interesa determinar la magnitud de estas desviaciones y sus valores relativos que resultan en particular de esta base. 
Hidrógeno

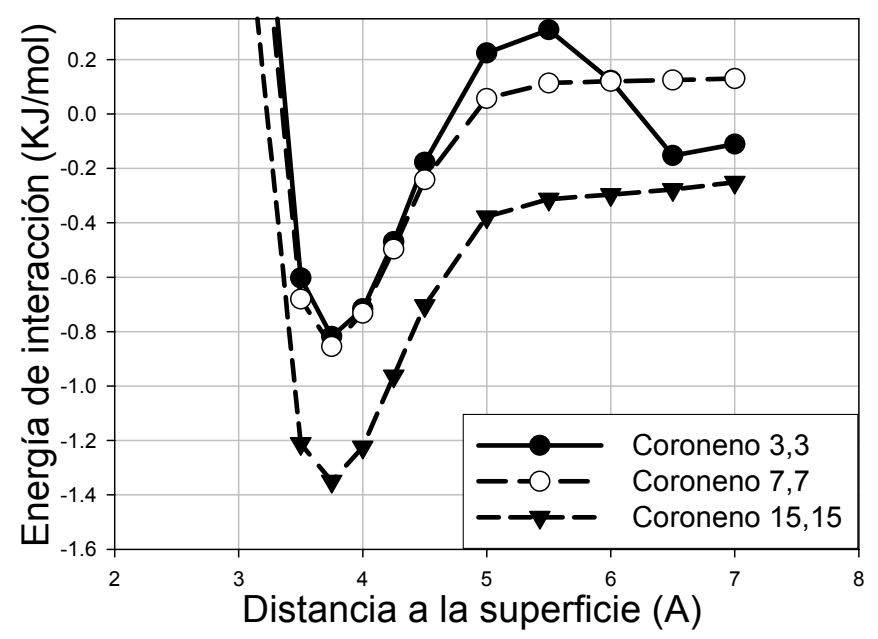

Fig 2: Curva de energía potencial para una molécula de hidrógeno

\section{Nitrogeno}

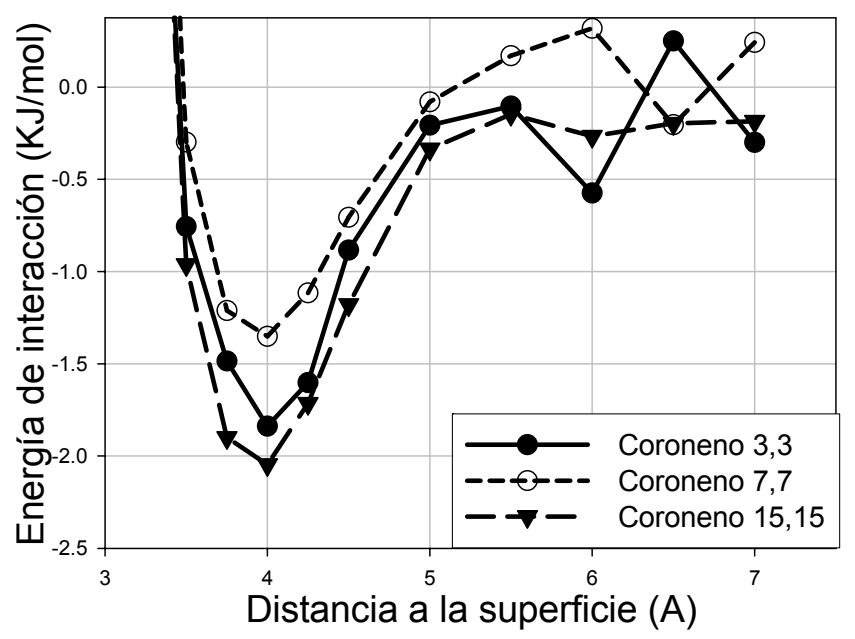

Fig 3: Curva de energía potencial para una molécula de nitrógeno

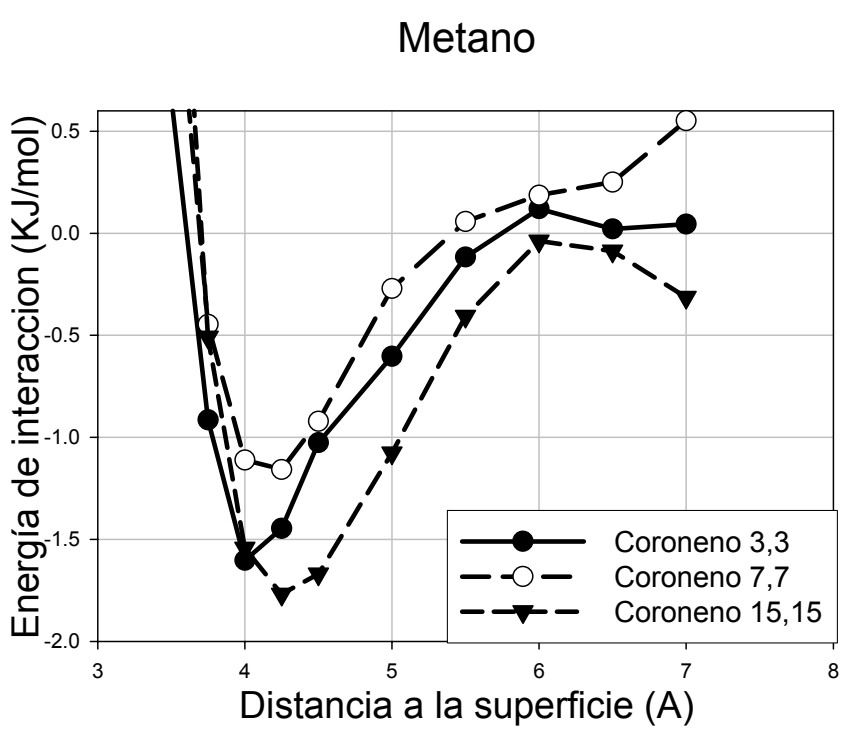

Fig 4: Curva de energía potencial para una molécula de metano 
Las fluctuaciones a distancias mas allá de 5 a 6 Ángstrom de la superficie se deben a que, a menos que se las incluya explícitamente (Girifalco y Hodak, 2002), DFT no toma en cuenta las interacciones de largo alcance, de todas maneras esto no afecta la presente descripción, pues la región de interés se encuentra alrededor del punto de equilibrio.

Las curvas fueron ajustadas a un potencial 12-6 de Lennard-Jones en la región alrededor del punto de equilibrio que no se extiende más allá de 5 Ángstrom por encima de la superficie. En la tabla 1 se muestran los parámetros resultantes del ajuste, allí también se incluyen los valores para grafito tomados de la literatura.

Tabla 1: Parámetros de Lennard-Jones obtenidos mediante el ajuste de las curvas

\begin{tabular}{ccccccc}
\hline & \multicolumn{2}{c}{ Nitrógeno } & \multicolumn{2}{c}{ Metano } & \multicolumn{2}{c}{ Hidrógeno } \\
\hline Nanotubo & $\sigma(\AA)$ & $\varepsilon(\mathrm{K})$ & $\sigma(\AA)$ & $\varepsilon(\mathrm{K})$ & $\sigma(\AA)$ & $\varepsilon(\mathrm{K})$ \\
\hline 3,3 & 3.70 & 20.19 & 4.02 & 14.73 & 3.76 & 7.41 \\
\hline 7,7 & 3.74 & 15.31 & 4.02 & 12.56 & 3.69 & 12.62 \\
\hline 15,15 & 3.68 & 18.77 & 4.18 & 14.91 & 3.68 & 9.36 \\
\hline Grafito & 3.60 & 44.71 & 3.61 & 64.37 & 3.18 & 32.06 \\
\hline
\end{tabular}

Se observa que la posición $(\sigma)$, del punto de cambio de signo del potencial, prácticamente no se modifica en estos nanotubos y la profundidad $(\varepsilon)$, del pozo de potencial, tampoco presenta una gran variación con la curvatura.

\section{CONCLUSIONES}

El análisis de los resultados obtenidos muestran que:

- En las curvas de energía potencial la posición del mínimo no se modifica con la curvatura de la superficie

- En todos los casos los valores de energía resultan inferiores a los obtenidos experimentalmente.

- La base adoptada da una buena descripción alrededor del punto de equilibrio.

- En regiones muy alejadas de la superficie la aproximación falla mostrando oscilaciones.

- Los parámetros que resultan de ajustar un potencial 12-6 de Lennard-Jones no muestran ninguna dependencia importante con la curvatura de los nanotubos en el rango considerado.

\section{AGRADECIMIENTOS}

A la UNLP (Universidad Nacional de La Plata), CICPBA (Comisión de Investigaciones Científicas de la Provincia de Buenos Aires) y CONICET (Consejo de Investigaciones Científicas y Tecnológicas), por el apoyo recibido.

\section{REFERENCIAS}

Albesa, A.G., J.L. Llanos y J.L. Vicente; Comparative Study of Methane Adsorption on Graphite, .Langmuir: 24(8), 3836 - 3840 (2008).

Albesa, A.G. y J.L. Vicente; Theoretical study of the adsorption of methane on graphite, J. Argent. Chem. Soc.: 95, 48-58 (2008).

Arora, G. y S. Sandler; Nanoporous carbon membranes for separation of nitrogen y oxygen: Insight from molecular simulations, Fluid Phase Equilibria: 259(1), 3-8 (2007).

Bhatia, S.K. y A.L. Myers; Optimum Conditions for Adsorptive Storage, Langmuir: 22(4), 1688-1700 (2006).

Boys, S.F. y F. Bernardi; The calculation of small molecular interactions by the differences of separate total energies. Some procedures with reduced errors, Molecular Physics: 19(4), 553-566 (1970). 
Cheng, H. y otros cinco autores; Molecular Dynamics Simulations on the Effects of Diameter $y$ Chirality on Hydrogen Adsorption in Single Walled Carbon Nanotubes, J. Phys. B: 109,3780-3786, (2005).

Colomer, J.F. y otros seis autores; Synthesis of single - wall carbon nanotubes by catalytic decomposition of hydrocarbons, Chem. Commun.: 9/03142A, 1343-1344 (1999).

Frisch, M. J. y otros 74 autores; Gaussian 03, Revision B.02, Gaussian, Inc., Pittsburgh PA, (2003).

Girifalco, L. A., y M. Hodak; Van der Waals binding energies in graphitic structures, Phys. Rev. B.: $65(12), 125404-1-5$ (2002).

Kostov, M. K., H. Cheng, A.C. Cooper y G.P. Pez; Influence of Carbon Curvature on Molecular Adsorptions in Carbon-Based Materials: A Force Field Approach, Phys. Rev. Lett.: 89 (14), 146105-14 (2002).

Niyogi, S. y otros 7 autores; Chemistry of Single-Walled Carbon Nanotubes ,Acc. Chem. Res., 35(12), $1105-1113,(2002)$

Okamoto, Y. e Y. Miyamoto; Ab Initio Investigation of Physisorption of Molecular Hydrogen on Planar y Curved Graphenes, J. Phys. Chem. B: 05(17), 3470-3474 (2001).

Talapatra, S. y A.D. Migone; Adsorption of methane on bundles of closed-ended single-wall carbon nanotubes, Phys. Rev. B: 65(4), 045416 (2002).

Vilaplana, A.F.; Ab initio computational investigation of physisorption of molecular hydrogen on achiral single - walled carbon nanotubes, J. Chem. Phys.: 122, 214724-1-7 (2005).

Wang, Q. y J. Johnson; Molecular simulation of hydrogen adsorption in single-walled carbon nanotubes y idealized carbon slit pores, J. Chem. Phys.: 110 (1), 577-586 (1999).

Wang, Y. y J.P. Perdew; Correlation hole of the spin-polarized electron gas, with exact small-wavevector y high-density scaling, Phys. Rev B: 44(24), 13298-13307 (1991).

Wei B.-Y. y otros 4 autores; Gases adsorption on single-walled carbon nanotubes measured by piezoelectric quartz crystal microbalance Materials Chemistry y Physics 81,126-133, (2003).

Zuttel, A.; Materials for hydrogen storage, Materials Today: 6(9), 24-33 (2003). 